\title{
Open Laryngeal Fracture: A case report and review of the literature
}

\author{
Burak Ulkumen $^{1 *}$, Onur Celik ${ }^{1}$, Nevin Sahin ${ }^{1}$
}

\begin{abstract}
Objectives: Laryngeal fracture is a rare and life threatening entity which is caused by blunt or penetrating neck trauma. Majority of laryngeal fractures are closed type and caused by blunt neck trauma. They are frequently left under diagnosed due to their closed nature and accompanying multisystem trauma. Also, open laryngeal fracture is exceptional and seen particularly in penetrating neck traumas like gunshot injuries. We describe an unusual case of open laryngeal fracture in which the patient was totally breathing from the wound as if a coniotomy had done.

Case: A 23-year-old male presented to the emergency department after getting involved in a motorcycle accident resulting with a blunt neck injury. The patient was breathing from a horizontal open neck wound below the level of the thyroid cartilage. Computed tomography revealed a laryngeal fracture involving thyroid, cricoid cartilages and the first ring of trachea. Given the extent of injury, a laryngeal reconstruction was performed. Six weeks after surgery he had no stridor, with evidence of full vocal cord mobility on both sides.

Conclusion: Early diagnosis is indispensable because it may cause acute airway compromise or significant long term squeal like dysphonia, aspiration, airway stenosis and tracheoesophageal fistula. In laryngeal fracture transoral intubation may lead irremediable results especially in the comminuted fractures or if there's arytenoid dislocation. Thus, in suspicion of laryngeal fracture associated with airway compromise one should always discuss tracheotomy rather than trans-oral intubation
\end{abstract}

Key words: Larynx, fracture, blunt, neck injury

\section{Introduction}

Laryngeal trauma (LT) is very rare (1-3) owing to elastic nature of larynx and protective effect of mandible and sternum. It has a reported incidence of 1 in 30000 patients among severe trauma admissions to the emergency department $(1,3)$. LT may range from simple mucosal tears to fractured and comminuted cartilage. If it is not thoroughly identified and properly treated LT may cause death due to airway compromise or may cause significant long term squeal like dysphonia, aspiration, and airway stenosis (4). Additionally, trans-oral intubation may lead catastrophic results like worsening the preexisting injury, causing further tears or cricotracheal separation.

Cervical and intracranial injuries are also common in these patients (5-9). For this reason, trauma guidelines concerning the potentially affected sites should also be considered. LT can be in 2 forms as penetrating or blunt. Penetrating injury commonly result from gunshots while blunt injury occurs mostly due to motorcycle accidents.

We report an extraordinary blunt neck trauma occurred during a motorcycle accident in which the patient was presented with breathing from the wound site as if a coniotomy had been done. The condition was due to a serious open laryngeal fracture including both the thyroid and cricoid cartilages associated with partial thyrocricoid separation. We provide a review of pertinent literature, with an emphasis on presentation to the emergency department, airway management, surgical reconstruction and classification of laryngeal traumas.

Case

A 23-year-old male presented to the emergency department after getting involved in a motorcycle accident resulting with a blunt injury of the neck. He was conscious but not able to talk. Upon initial ENT evaluation, it was noted that the patient was breathing from a horizontal open neck wound as if a coniotomy had been done (Figure 1).

Borders of the horizontal wound were clean with a length of 3 centimeters and there was no sign of any massive hemorrhage. Flexible laryngoscopic examination revealed paralyses of both vocal cords. There were also a considerable amount of edema and ecchymosis at both false vocal cords and aryepiglottic folds. After acute stabilization, the patient underwent computed tomography (CT) scan, revealing a laryngeal fracture involving thyroid, cricoid and the first ring of trachea.

Non comminuted fractures were also determined in the level of 5th, 6th and 7 th cervical vertebra. 

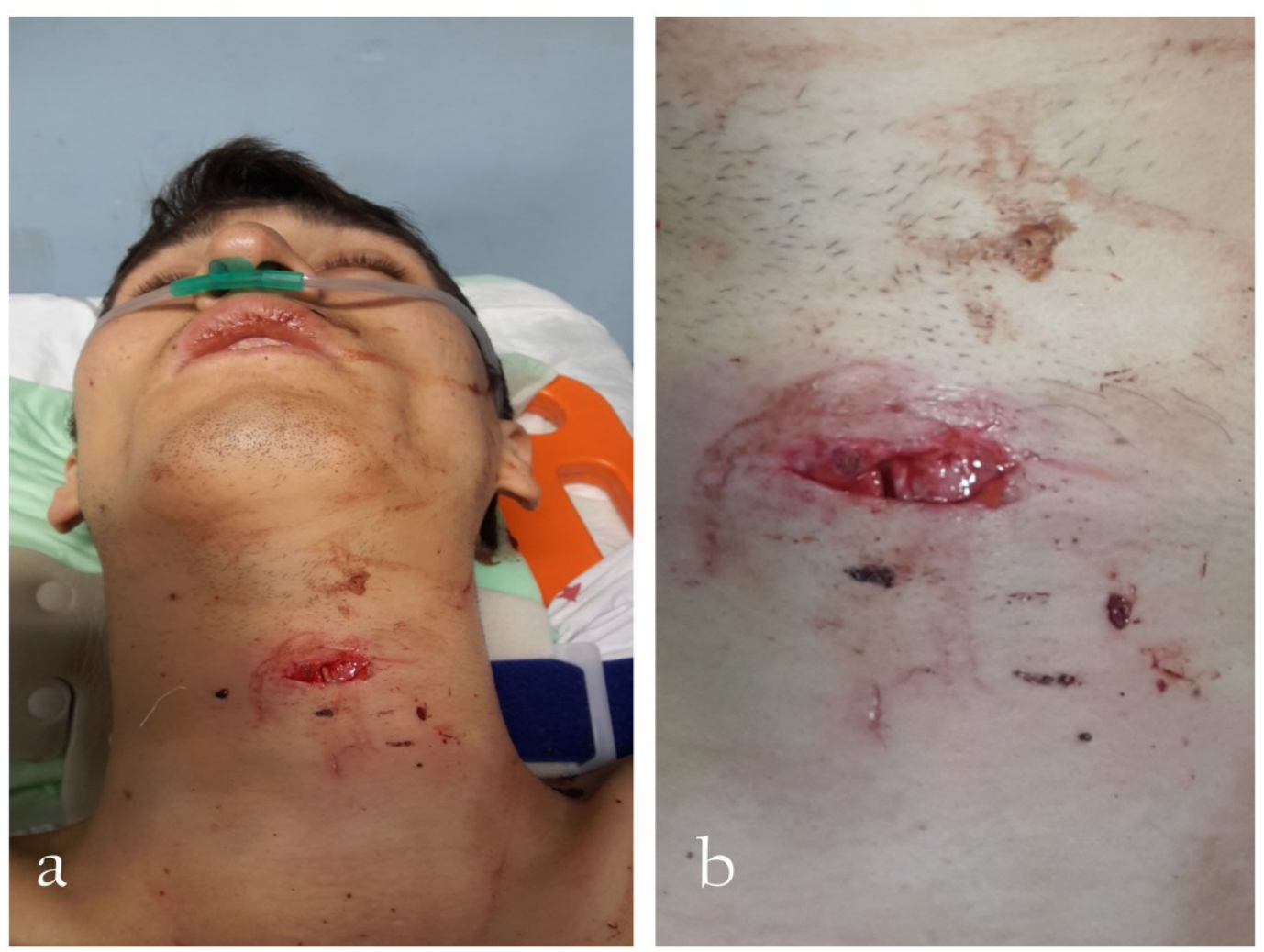

Figure 1 (a) Horizontal wound at the level of Thyroid Cartilage. (b) Closed view showing fracture line on the thyroid cartilage in relationship with the wound

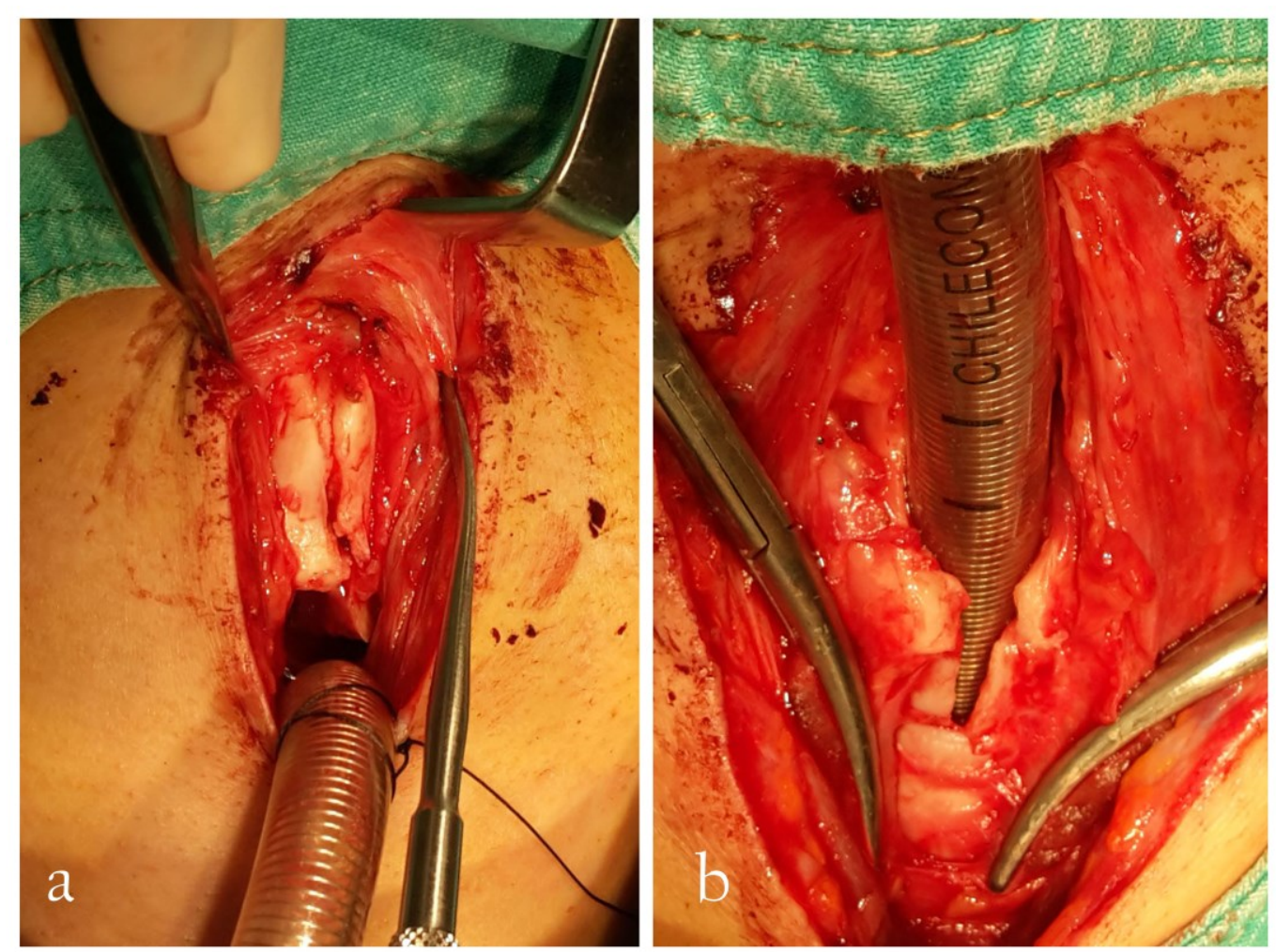

Figure 2 (a) Thyroid cartilage fracture line is seen while the intubation tube is oriented superiorly. (b) Fracture line involving the cricoid and first ring of trachea is seen while the intubation tube oriented inferiorly 
Neurological examination was normal except a minimal numbness at the right hand and forearm. Neurosurgical department did not suggest any surgical intervention. They applied only a cervical collar.

In the light of these findings we decided to perform a surgical exploration. The patient was intubated through the open wound and exploration was carried out via this open neck wound with the endotracheal tube in view. It was seen that there was a clean fracture line starting from the thyroid notch including the whole anterior wall of the thyroid and cricoid cartilages, then extending inferiorly down to the inferior edge of first tracheal ring (Figure 2).

The cricothyroid membrane and anterior mucosa of subglottic region was totally defective leading a partial separation of thyroid and cricoid cartilages. After first evaluation, a 2nd vertical skin incision, starting from the wound, was performed and a tracheotomy between the 3rd and 4th tracheal ring was carried out. Then the endotracheal tube was replaced inferiorly to this tracheotomy site for better visualization of the posterior wall of subglottic area and cricoid cartilage. After replacement of the endotracheal tube it was noted that the subglottic mucosa was ripped and peeled off the posterior wall of the cricoid cartilage. A non-displaced fracture line was also noted on the stripped posterior wall of cricoid cartilage. Besides, the cricoarytenoid joint of both sides were intact.

Given the extent of injury, a thyrocricoidal reconstruction was attempted. Firstly, we started to the reconstruction with the repair of subglottic mucosa from posterior to anterior. The teared superior and inferior edges of posterior subglottic mucosa were approximated and sutured by using 5-0 Vicryl. Then the laryngeal fractures were reduced and fixated using 2-0 Prolene sutures. Attention was then devoted to the management of partial thyrocricoidal separation. Using 3-0 Vicryl, the thyroid cartilage was sutured anterolaterally to the cricoid cartilage. These sutures were loosely tied to help re-approximate the airway. Two 2-0 Prolene stay sutures were placed through the thyroid cartilage and the 1 st tracheal ring to secure this repair.

The patient's postoperative course was uncomplicated. The patient was observed in the intensive care unit for 2 days and then total parenteral nutrition was started. At the second day of surgery a barium esophagogram was done which was suspicious about leakage. So an esophagoscopy was also done demonstrating no evidence of an esophageal disruption.

Oral intake was started approximately 4 weeks after injury. The patient showed signs of vocal cord recovery 6 weeks after the injury on both sides, and the tracheostomy was closed. On last follow-up (3 months after the injury), he had no stridor, with evidence of full vocal cord mobility on both sides.

\section{Discussion}

Laryngeal framework is protected superiorly by maxilla and mandibula, inferiorly by sternum. Due to this protective effect and elastic nature of larynx, laryngeal fracture is very rare (1-3). It is rare also because severe laryngeal trauma patients die be $\neg$ fore reaching a medical facility owing to serious airway compromise or associated multisystem trauma. Thus, a considerable amount of laryngeal fracture cases become under-diagnosed $(2,3)$.

Laryngeal fracture can be caused by 2 mechanisms as blunt neck trauma or penetrating neck trauma. Blunt neck trauma almost always occurs in connection with motor vehicle accidents, and it has been reported to be the most frequent cause (10). But in recent years it has a decreasing tendency due to improvement of airbags and abun $\neg$ dant safety devices for riders (11). Besides, blunt trauma related laryngeal fracture due to sportive events in which motorcycles, bicycles, skis, etc. are used is on increase (12).

Penetrating neck trauma is relatively rare. In the past, gunshots were the leading causes. But, nowadays owing to industrialization and urbanization penetrating trauma is also on increase because of machines related accidents and stabbing (10). Laryngeal trauma patients may present with hoarse $\neg$ ness, stridor, respiratory distress, subcutaneous emphysema, hemop $\neg$ tysis, hematomas, subcutaneous hemorrhage, laryngeal tenderness, vocal cord dysfunction, and the loss of an anatomical landmark in the neck $(1,13)$. Our case was a blunt neck trauma due to a motorcycle accident in which the patient hit a lamppost.

The type of injury was pertinent with the literature but the presentation was very exceptional. At the admission, the patient was totally breathing from a midline horizontal open wound at the level of the cricothyroid membrane as if a cricothyrotomy (coniotomy) was done (Figure 1). There was no respiratory distress.

Esophageal injury may also occur in LT cases. Hence, barium esophagogram and/or esophagoscopy should be done to rule out esophageal disruption $(3,14,15)$. In our case the barium esophagogram was suspicious but the esophagoscopy was normal. So the patient able to fed trans-orally. Vocal cord paralyses are also common in laryngeal fracture cases and may occur due to direct injury or secondary injury caused by edema or hematoma. In the present case the paralyses recover at the 6th week so the probable mechanism was vocal cord injury secondary hematoma and edema.

Open fractures of the laryngeal framework are quite uncommon, and likely to be missed only by failure to examine the neck, which may perhaps be obscured by a cervical collar as in our case. On the contrary, fractures of the larynx are more likely to be "open" internally, where the defect may be through the mucosa rather than the skin, and therefore missed 
by external examination alone. These injuries may ultimately require endoscopic or open repair, or tracheotomy (16).

In our case there was an open wound through which the fractured thyroid and cricoid cartilage were seen by close observation (Figure 1). This type of presentation is very uncommon for a blunt neck injury. We think that this is caused by high torsional forces leading the neck to over-extension during the accident. This also may be the liable mechanism explaining the cervical fractures in the case reported.

Schaefer had classified the laryngeal injuries into $\mathrm{V}$ groups according to the severity (1). Our case was compatible with group IV which is defined as having disruption of anterior larynx, unstable fractures, two or more fractures lines, or severe mucosal injuries. Not all the laryngeal trauma patients need surgical intervention. Group-I injuries can be managed conservatively. Group II to IV patients commonly necessitates surgical intervention as endoscopic, open or both. In the present case open surgical repair was inevitable.

Additionally, in this classification system the condition of the trachea had not been taken into account except the group $\mathrm{V}$ which is defined as complete laryngotracheal separation. However, concerning the concurrent tracheal injury in laryngeal trauma patients there should be a more detailed definition. For example in our case there was a midline cut of first tracheal ring leading a partial laryngotracheal separation which is not defined in the aforementioned classification. Thus, we think that a more precise classification should be defined.

In the literature, 3 different external techniques for the reduction of LF were defined. These are make use of miniplates, wire or primary suturation for reconstruction of the laryngeal skeleton $(14,16,17)$. We preferred to use primary suturation because there are some reports about fibrous healing especially with wire usage. Concerning the miniplate usage; it is more suitable in comminuted or severely displaced fractures.

Consequently, open laryngeal fracture following blunt neck trauma is very rare and the reported cases, unlike our case, were internally open fractures due to torn laryngeal mucosa. Additionally, totally breathing from the wound is a very exceptional presentation. Emergency physician should avoid intubation in suspicion of laryngeal trauma otherwise it may worsen the preexisting injury and may cause further tears or even cricotracheal separation leading long term complications like dysphonia, aspiration, and airway stenosis.

Conflict of Interest: The authors declare no potential conflicts of interest with respect to the research, authorship, and/or publication of this article.

\section{References}

1. Schaefer SD. The acute management of external laryngeal trauma. A 27-year experience. Arch Otolaryngol Head Neck Surg. 1992 Jun;118(6):598-604

2. Gussack GS, Jurkovich GJ, Luterman A. Laryngotracheal trauma: a protocol approach to a rare injury. Laryngoscope 1986 Jun;96(6):660-5

3. Jalisi S, Zoccoli M. Management of laryngeal fractures--a 10year experience. J Voice. 2011 Jul;25(4):473-9.

4. Mendelsohn AH, Sidell DR, Berke GS, John MS. Optimal timing of surgical intervention following adult laryngeal trauma. Laryngoscope. 2011 Oct;121(10):2122-7

5. Butler AP, Wood BP, O'Rourke AK, Porubsky ES. Acute external laryngeal trauma: experience with 112 patients. Ann Otol Rhinol Laryngol. 2005 May;114(5):361-8.

6. Yen PT, Lee HY, Tsai MH, Chan ST, Huang TS. Clinical analysis of external laryngeal trauma. J Laryngol Otol. 1994 Mar;108(3):221-5.

7. Harris $\mathrm{HH}$, Tobin HA. Acute injuries of the larynx and trachea in 49 patients. (Observations over a 15 -year period). Laryngoscope. 1970 Sep;80(9):1376-84

8. Leopold DA. Laryngeal trauma. A historical comparison of treatment methods. Arch Otolaryngol. 1983 Feb;109(2):10612

9. Schild JA, Denneny EC. Evaluation and treatment of acute laryngeal fractures. Head Neck. 1989 Nov-Dec;11(6):491-6.

10. Kim JP, Cho SJ, Son HY, Park JJ, Woo SH. Analysis of clinical feature and management of laryngeal fracture: recent 22 case review. Yonsei Med J. 2012 Sep;53(5):992-8.

11. Irish JC, Hekkenberg R, Gullane PJ, Brown DH, Rotstein LE, Neligan P, Ali J. Penetrating and blunt neck trauma: 10-year review of a Canadian experience. Can J Surg. 1997 Feb;40(1):33-8

12. Lee WT, Eliashar R, Eliachar I. Acute external laryngotracheal trauma: diagnosis and management. Ear Nose Throat J. 2006 Mar;85(3):179-84.

13. Hwang SY, Yeak SC. Management dilemmas in laryngeal trauma. J Laryngol Otol. 2004 May;118(5):325-8.

14. Bent JP 3rd, Silver JR, Porubsky ES. Acute laryngeal trauma: a review of 77 patients. Otolaryngol Head Neck Surg. 1993 Sep;109(3 Pt 1):441-9.

15. Vassiliu P, Baker J, Henderson S, Alo K, Velmahos G, Demetriades D. Aerodigestive injuries of the neck. Am Surg. 2001 Jan;67(1):75-9.

16. Chitose S, Sato K, Nakazono H, Fukahori M, Umeno H, Nakashima T. Surgical management for isolated cricoid fracture causing arytenoid immobility. Auris Nasus Larynx. 2014 Apr;41(2):225-8.

17. Thor A, Linder A. Repair of a laryngeal fracture using miniplates. Int J Oral Maxillofac Surg. 2007 Aug;36(8):748-50.

Copyright (C) 2014 The Author(s); This is an open-access article distributed under the terms of the Creative Commons Attribution License (http://creativecommons.org/licenses/by/4.0), which permits unrestricted use, distribution, and reproduction in any medium, provided the original work is properly cited. All Rights reserved by international journal of Medical Science and Discovery and Lycians Press Inc. 